\title{
Zwei Seelen wohnen, ach! in meiner Brust ...
}

\author{
Digitalität und Sexualität in der Sozialen Arbeit
}

Digitalität und Sexualität sind - insbesondere in ihrer Verwobenheit - gesellschaftlichen Wandlungsprozessen unterlegen. In ihrer alltagsweltlichen Durchdringung fordern diese Prozesse Heranwachsenden ebenso wie pädagogischen Fachkräften eine Auseinandersetzung ab, die sich als sexualbezogene Medienkompetenz beschreiben lässt. Welche Herausforderungen ergeben sich aus diesen Anforderungen und aus den möglicherweise damit verbundenen Problemen für die Soziale Arbeit?

$\mathrm{N}$ eben den nach wie vor stark genutzten sozialen Medienplattformen wie YouTube, Twitter, Instagram und Facebook, erfreut sich die Videoplattform TikTok seit 2018 gerade bei Jugendlichen und jungen Erwachsenen großer Beliebtheit und avanciert im deutschen und englischsprachigen Raum insbesondere auch bei LSBTQ-Jugendlichen zu einem Ort, an dem sie sich mit ihrer Sexualität und vergeschlechtlichten Positionierung ausprobieren können. „Tiktok“ so die dieser Einsicht Rechnung tragende Formulierung

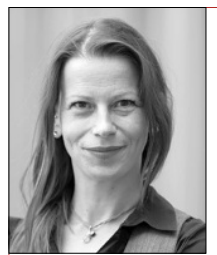

\section{Alexandra Klein}

Johannes Gutenberg-Universität Mainz, Mainz, Deutschland

*1975, Dipl.-Päd., Dr. phil. Professorin für Erziehungswissenschaft im Schwerpunkt Heterogenität und Diversität am Institut für Erziehungswissenschaft, AG Sozialpädagogik der Johannes Gutenberg-Universität Mainz. Arbeitsschwerpunkte: Heterogenität und Ungleichheiten in Kindheit und Jugend, Interventionslogiken Sozialer Arbeit sowie quantitative und qualitative Unterstützungsforschung.

alexandra.klein@uni-mainz.de

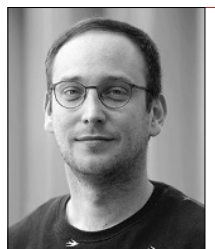

\section{Jann Schweitzer}

Johannes Gutenberg-Universität Mainz, Mainz, Deutschland *1986, Erziehungswissenschaften M.A.; wissenschaftlicher Mitarbeiter am Institut für Erziehungswissenschaft, AG Sozialpädagogik der Johannes Gutenberg-Universität Mainz. Arbeitsschwerpunkt Theorie und Empirie von Ungleichheit im Jugendalter, schulische Sexualerziehung und sexualitätsbezogene Bildungsforschung.

jannschweitzer@uni-mainz.de

Zusammenfassung Der Beitrag thematisiert die Mediensozialisation von Heranwachsenden im Zusammenhang mit Sexualität. Nach der Präsentation verschiedener empirischer Befunde zur Mediennutzung und zur Sexualität fokussiert er zunächst die Phänomene Pornografienutzung und Sexting, um daraus dann Anschlusspunkte für die Soziale Arbeit darzustellen.

Schlüsselwörter Sexting, Digitalität, Sexualität, TikTok, Mediennutzung, Pornografie von Abby Ohlheiser "has become the soul of the lgbtq internet" (Ohlheiser 2020, o. S.).

Obwohl sich die Plattform in diesem Kontext durchaus als jung und subversiv präsentiert, zeigen viele Inhalte auch ein deutlich anderes Bild. So finden sich eine Vielzahl von Beiträgen, in denen gerade männliche Nutzer etwa unter dem Titel „Die 3 größten Fehler, die Frauen beim Daten machen " Ratschläge und (vermeintliche) Erfahrungen verbreiten, die mit Blick auf ihre sexistischen und heteronormativen Inhalte problemlos den 1950er-Jahren entstammen könnten. Weiter berichten viele Nutzer_innen von übergriffigen Erfahrungen, von sexualisiertem Cyber-Mobbing und der unerlaubten Weitergabe und Verbreitung eigener Bilder und Videos (Vogelsang 2017, S. 278). Solcherart problematische Erfahrungen sind es, die gegenwärtig im Rahmen medienpädagogischer Präventionsprogramme bearbeitet und aufgegriffen werden. Gleichzeitig offenbaren diese Ansätze bei genauerer Betrachtung mitunter sexualmoralisch fragwürdige Interpretationen, die die Probleme in diesem Kontext vorrangig in einer vermeintlich „freizügigen Selbstdarstellung“ (Rack und Sauer 2020, S. 24 ff.) seitens der Jugendlichen verorten.

Fernab dieser Kritik bewahrpädagogischer Präventionsprogramme verdeutlicht ein (sozial-)pädagogischer Blick auf das Spannungsfeld im Nutzungsverhalten auf TikTok die hohe Relevanz eines eher unzureichend aktualisierten Themas im Rahmen erziehungs- und sozialwissenschaftlicher Auseinandersetzung, nämlich die zentrale Bedeutung einer digitalen Medienkompetenz als Entwicklungsaufgabe Heranwachsender sowie gleichzeitig die professionelle Kompetenzanforderung an pädagogische Fachkräfte vor dem Hintergrund sich ständig wandelnder Prozesse der „Ökonomisierung, Kommerzialisierung, Digitalisierung und damit verbundenen Hybridisierungen von Privatheit und Öffentlich- 
keit“ (Paus-Hasebrink 2020, S. 1229). In besonderer Weise ist hier die sexualitätsbezogene Kommunikation mittels digitaler Medien betroffen, da digitale Räume zwar in erster Linie große Potenziale für eine erweiterte Auseinandersetzung mit der eigenen Sexualität eröffnen (Klein 2014), damit gleichzeitig aber auch erweiterte Gefahren von sexuellen Grenzverletzungen und Viktimisierungen (Klein 2017; Böhm und Budde 2020) relevant werden können. Ohne dabei auf moralpanische Sexualdiskurse der letzten Jahrzehnte einer „Generation Porno“ (Gernert 2010) hereinzufallen, betrachtet dieser Beitrag die durchaus spannungsreichen Herausforderungen in der Verwobenheit von Digitalität und Sexualität zunächst für die sexualbezogene Mediensozialisation von Heranwachsenden, indem die empirische Ausgangslage zur Mediennutzung und zur Sexualität skizziert wird, um ausgehend davon Anschlusspunkte an die Soziale Arbeit herzustellen.

\section{Mediennutzung von Heranwachsenden}

Aus dem Alltag von Heranwachsenden sind digitale Medien nicht mehr wegzudenken. Mit Blick auf die aktuellen Repräsentativdaten der JIM-Studie (mpfs 2020) zum Medienumgang von 12- bis 19-jährigen Jugendlichen und jungen Erwachsenen bestätigt sich diese Allgegenwärtigkeit in Bezug auf den Zugang bzw. den Gerätebesitz der Jugendlichen. $94 \%$ der Jugendlichen haben ein eigenes Smartphone. Auch Computer oder Notebooks sind mit $72 \%$ bei fast drei Viertel der Jugendlichen im eigenen Zimmer zu finden (ebd, S. 8). Diese Geräte werden in erster Linie zur Unterhaltung (34 \%) genutzt. Fast gleichauf liegen die Bereiche Kommunikation $(27 \%)$ und Spiele spielen $(28 \%)$. Den kleinsten Bereich bildet die Informationssuche mit elf Prozent. Im Vergleich zu den früheren Untersuchungen (vgl. mpfs 2015) fällt der steigende Anteil der Nutzung zu Unterhaltungszwecken und der kleiner werdende Anteil der Kommunikation auf. Während vor fünf Jahren noch die Kommunikation der bedeutendste Bereich der Internetnutzung Heranwachsender war, lässt sich gegenwärtig eine Annäherung und Überschneidung der Bereiche Kommunikation, Spiele und Unterhaltung feststellen (mpfs 2020, S. 34). Gleichzeitig scheint hier Differenzierung notwendig. Denn wird nach der Bedeutung digitaler Angebote bzw. nach der Wichtigkeit von verschiedenen Applikationen (Apps) gefragt, geben $82 \%$ der Jugendlichen WhatsApp und etwa die Hälfte Instagram an (ebd., S. 37). Die bedeutsamsten Apps sind demnach Kommunikationsplattformen, in denen Fotos, Videos und Nachrichten geteilt, geliked und privat verschickt werden und gleichsam zur eigenen Unterhaltung, aber auch für eine kleinere oder größere
Öffentlichkeit („Freund*innen/Follower*innen“) dienen können.

Solche Apps lassen sich nun aus einer sexualitätsbezogenen Perspektive als potenzielle Gelegenheitsräume verstehen, die Möglichkeiten für sexuelle, soziale und romantische Beziehungen, für Erfahrungen mit Pornografie, (erste) sexuelle Erfahrungen, aber auch der Klärung sexualbezogener Fragen bieten können und auch in diesem Sinne von den Heranwachsenden genutzt werden. Gleichzeitig können diese vielgestaltigen inhaltlichen Nutzungsmöglichkeiten eben auch von gewaltvollen und grenzverletzenden sexualitätsbezogenen Erfahrungen begleitet werden. Sexuelle Kommunikationen und Interaktionen sind somit im Lebensalltag von Heranwachsenden eng mit Digitalität bzw. den Möglichkeiten und Formen digitaler Räume verknüpft. Dabei erweisen sich mit Blick auf die Entwicklung einer sexualbezogenen Medienkompetenz von vor allem zwei Nutzungsaspekte als aufschlussreich, nämlich die Pornografienutzung und das Phänomen des Sexting, wenn es um die Frage geht, wie Jugendliche digitale Räume im Rahmen ihrer sexuellen Sozialisation nutzen.

\section{Sexualität und digitalen Medien}

Pornografische Bilder und Filme waren noch nie so leicht zugänglich wie heute. Das Internet eröffnet einen schier grenzenlosen Fundus an vielfältigem und explizit sexuellem Material. Unter Heranwachsenden ist die Erfahrung damit seit Jahren konstant hoch. Fast jede_r zweite Jugendliche im Alter zwischen 13 und 18 Jahren hat im Jahr 2016 bereits einmal Kontakt zu pornografischen Inhalten gehabt (Bauer Media Group 2016). Die für die Bundesrepublik umfangreichsten Zahlen bezüglich entsprechender Erfahrungen liefert die medienwissenschaftliche Repräsentativstudie von Thorsten Quandt und Jens Vogelgesang (2018). Danach verfügt knapp die Hälfte der befragten Jugendlichen über Erfahrungen mit pornografischen Filmen oder Bildern. Hauptsächlich über mobile Endgeräte wie Smartphones oder Laptops werden dabei kurze Videoclips auf Streaming-Plattformen genutzt. Jungen zeigen sich in dieser Hinsicht erfahrener als Mädchen, sie nutzen Pornografie häufiger und intensiver (ebd., S. 106). Gleichaltrige bilden dabei sowohl für Jungen wie auch für Mädchen einen bedeutsamen Nutzungskontext: Während die Hälfte der Jugendlichen angab, beim ersten Kontakt mit pornografischem Material alleine gewesen zu sein, erfolgte bei rund $40 \%$ der Befragten der Erstkontakt zusammen mit Peers (ebd., S. 108). Peer-Beziehung bieten sind demnach potenzielle Gelegenheitsräume; sowohl für sexuelle Beziehungen als auch für Erfahrungen mit Pornografie. Die Konfrontation mit bzw. Nutzung 


\section{Extrablick: Sexualität in der Sozialen Arbeit}

von pornografischen Inhalten - seien sie gewollt oder ungewollt - kann mit neuen und zusätzlichen Fragen, Irritationen und Sorgen einhergehen. Sie bilden gleichsam einen Anlass für Reflexionen, die für das Jugendalter ganz grundsätzlich charakteristisch sind: Unsicherheiten über das Körperselbstbild und die eigene Attraktivität, Unsicherheit über die eigene Sexualität und den ,richtigen' Umgang mit Partner_innen oder Sorgen darüber, einen passenden Lebensentwurf für sich selbst zu finden und zu verwirklichen. Es ist durchaus denkbar, dass solche Verunsicherungen durch pornografische Inhalte verstärkt werden.

Gleichzeitig spricht auch einiges dafür, Pornografie als Kommunikationsanlass zu verstehen, der sowohl Auseinandersetzung als auch Distanzierung und Unterstützung ermöglicht. So wird bereits der erste Kontakt mit Pornografie von einem Drittel der befragten Jugendlichen mit anderen besprochen, hauptsächlich mit Freund_innen (ebd., S. 112). Nutzer_innen, die sich von den pornografischen Inhalten erregt fühlten, sprechen dabei am seltensten über ihre Nutzung. Dies kann als Hinweis „,auf die anhaltende Tabuisierung des Themas“ (ebd., S. 114) gelesen werden. Diejenigen, die sich dabei etwa geekelt oder unwohl gefühlt haben, sprechen demgegenüber etwas häufiger über ihre Erfahrungen. Gleichzeitig heben die wenigen qualitativen Analysen insbesondere die soziale Dimension der Nutzung und des Erlebens von Pornografie hervor. Mit den unterschiedlichen sozialen Settings, ob alleine, mit Partner_innen, in einer Mädchen- oder Jungenclique oder etwa auf einer Party oder im Jugendhaus, gehen unterschiedliche Nutzungsmotive und -möglichkeiten einher (Matthiesen 2014). Wird Pornografie in der öffentlichen Diskussion nahezu ausschließlich mit sexueller Erregung assoziiert, so handelt es sich hierbei zwar um eine relevante, keinesfalls jedoch um die ausschließliche Nutzungsweise Heranwachsender. Vielmehr ist die Nutzung mit einem breiten Spektrum sozialer und kompetenzbezogener Motivationen verbunden: Es geht um (gemeinsame) Belustigung, um Abgrenzung und $\mathrm{Zu}-$ gehörigkeit, um Tabubrüche und Grenzverschiebungen genauso wie um die Demonstration sexueller Kompetenzen und Erfahrenheit, sowie die Erarbeitung sexualitätsbezogenen Wissens und das Ringen um sexuelle Handlungsfähigkeit (vgl. auch ebd.; Klein 2017).

Ähnlich unterschiedliche Nutzungsweisen lassen sich auch beim Phänomen des Sexting identifizieren, das ganz grundlegend den Austausch persönlicher sexueller Themen mittels digitaler Kommunikationsmedien beschreibt. Eine quantitative Befragung von Verena Vogelsang (2017) zeigt, dass Sexting von einem nicht unerheblichen Teil der befragten Jugendlichen zwischen 14 und 18 Jahren als Form sexueller Kommunikation genutzt wird. Knapp ein Drittel der Befragten gab demnach an, bereits eigene explizite Bilder oder Videos verschickt zu haben (ebd., S. 273) und $55 \%$, schon eine oder mehrere Sexting-Nachrichten bekommen zu haben (ebd.). Dabei sind die Adressat_innen fast ausschließlich die zu diesem Zeitpunkt aktuellen Partner_innen derjenigen, die sie verschicken. Die Motivationen, solche Nachrichten $\mathrm{zu}$ versenden, sind vielfältig. So verschickt ein Großteil der befragten männlichen Jugendlichen, nämlich $53 \%$, mit der Intention „zu flirten“, während die Hauptmotivation bei den weiblichen Befragten mit $43 \%$ als „sexy Geschenk “ verschickt wurden (ebd., S. 276). Darüber hinaus erweisen sich auch Versuche der Selbstinszenierung und Selbstbestätigung sowie insgesamt das Interesse daran, solche sexualitätsbezogenen Kommunikationsweisen ausprobieren, als nutzungsrelevant.

Gleichzeitig gibt auch ein Zehntel der Befragten an, dass Fotos oder Videos ohne ihre Zustimmung weitergeleitet wurden und knapp ein Viertel berichtet, bereits Fotos und/oder Videos anderer ohne Einverständnis weitergeleitet zu haben. Eine deutliche Mehrheit von $67 \%$ der Befragten vertritt darüber hinaus die Auffassung, dass, wer intime Fotos von sich verschicke, selbst schuld sei, wenn die Bilder dann an die Öffentlichkeit gelängen (vgl. Vogelsang 2017, S. 287). Diese Form der Täter-Opfer-Umkehr bei der unerlaubten Weitergabe von Chats, Bildern oder Videos hängt dabei im besonderen Maß mit sexistischen Zuschreibungen zusammen und trifft vor allem Mädchen und junge Frauen. Solcherart Grenzüberschreitungen widmet sich das qualitative Forschungsprojekt "Safer Sexting“ (Böhm und Budde 2020), das auf die Rekonstruktion von Zusammenhängen zwischen digitalen Medien und Grenzverletzungen anhand von Gruppendiskussionen zielt. Dabei berichten die diskutierenden Jugendlichen im Alten von 16 und 17 Jahren von ihren Erfahrungen beim Erhalten, Weiterleiten und Posten von sexuell expliziten Bildern und Videos. Sie berichten dabei von Cybermobbing und sog. ,Slut Shaming', also der Abwertung des vermeintlichen oder realen Sexualverhaltens von Mädchen* und Frauen*, sowie der bereits benannten Täter-Opfer-Umkehr, die sämtlich durch vergeschlechtlichte Zuschreibungen markiert sind (ebd., S. 16). Als zentrales Ergebnis halten sie fest, dass die Erzählungen der Jugendlichen insgesamt zwischen Devianz- und Normalitätsdiskursen zu verorten seien. „Einerseits wird das Risiko des Versendens und Postens sexuell expliziter Bilder benannt, aber individualisiert und damit als Verantwortung der jeweiligen Jugendlichen markiert, andererseits erscheint nicht nur das Zirkulieren dieser Bilder als ,Normalität', sondern auch die geschlechterbezogen differenten Erfahrungen und Umgangsweisen. Als besonders , selbst- 
bestimmt, konsensuell und wechselseitig' tritt Sexting dabei ebenso wenig auf wie als herausragendes Risiko“ (ebd.). Digitale Medien sind demnach mittlerweile in den Lebensrealitäten Heranwachsender zu einem selbstverständlichen Teil sexueller Sozialisation avanciert, in dem sich sexuelle Grenzverletzungs- und Selbstbestimmungspotenziale amalgamieren.

\section{Herausforderungen für die Soziale Arbeit}

Genau diese Ambivalenzen in der alltagsweltlichen Durchdringung von Sexualität und Digitalität stellen zentrale Herausforderungen an die Medienkompetenzentwicklung nicht nur für Heranwachsende, sondern auch für pädagogische Fachkräfte dar. Fachkräften Sozialer Arbeit kommt die Aufgabe zu, geeignete Angebote zu schaffen, die im Anschluss an die fachliche Reflexion von alltagsweltlichen Erfahrungen und Bezügen ihrer Adressat_innen auf die Eröffnung und Erweiterung von Teilhabechancen und Handlungsspielräumen zielen. Vor diesem Hintergrund erweisen sich die von Friederike Siller et al. (2020) herausgearbeiteten fachlichen Wissensdimensionen von zentraler Bedeutung. Dabei geht es für die Fachkräfte um die Erarbeitung von Orientierungswissen über digitale Entwicklungen und deren Verquickung mit sozialen und kulturellen Wandlungsprozessen, um Fachwissen über die Auswirkungen und Herausforderungen auf die Lebenswelten und Lebenslagen von Adressat_innen und um die Reflexion der eigenen Medienkompetenz und dem persönlichen Umgang damit. Diese reflexiven medienpädagogischen Analyse- und Gestaltungsfähigkeiten erweisen sich als elementare Voraussetzungen für die Gestaltung von medialen Bildungsräumen zur Förderung von Medienkompetenz (ebd., S. 327).

Pädagogische Fachkräfte stehen dementsprechend bereits vor dem Hintergrund gesellschaftlicher Mediatisierungsprozesse vor der ständigen Herausforderung „eigene Kommunikationsroutinen zu überwinden und den individuellen Handlungs- und Orientierungsspielraum durch das Medienhandeln zu erweitern“ (ebd., S. 322). In der Verwobenheit von Digitalität und Sexualität erweist sich darüber hinaus Nicola Dörings (2015) befähigungsorientierter Ansatz eines „Safer Sexting“ als anschlussfähig. Dieser orientiert sich an der Idee der Pornografie-Kompetenz (Döring 2011) und kann insgesamt als ein Entwurf sexualitätsbezogener Medienkompetenz beschrieben werden. So hat Döring ein mehrdimensionales Modell herausgearbeitet, das u. a. folgende Komponenten enthält:

1. Medienkunde als Kenntnisse über Produktion, Merkmale, Inhalte und Nutzung von Pornografie,

2. Kritikfähigkeit als Auseinandersetzung mit den potenziellen Negativwirkungen von Pornografie,
3. Genussfähigkeit als Auseinandersetzung mit den potenziellen Positivwirkungen von Pornografie,

4. die Fähigkeit zur Metakommunikation als konstruktiver Austausch über Pornografie und schließlich

5. die Fähigkeit zur Selbstreflexion als Reflexion des eigenen Standpunkts zur Pornografie (ebd., S. 240).

Damit liegt eine Heuristik vor, die eine Orientierung für die pädagogische Auseinandersetzung mit der ambivalenten Verwobenheit von Digitalität und Sexualität bieten kann. Werden etwa in einer zielgruppensensiblen Weiterführung dieser Heuristik sexualisierte Macht- und Gewaltverhältnisse systematisch mitgedacht, kann es gelingen, sexuelle Grenzverletzungen ebenso in den Blick zu bekommen, wie die sexuellen Selbstbestimmungspotenziale, die digitale Medien ihren Nutzer_innen offerieren. In diesem Sinne kann gerade auch die potenzielle Kompliz_innenschaft stigmatisierender und responsibilisierender Zuschreibungen der vermeintlich selbstverschuldeten und freizügigen Nutzungspraxis selbst zum Gegenstand professioneller Reflexion werden. Dies erweist sich als umso bedeutsamer, wie solche Zuschreibungen, die durch Fachkräfte ebenso wie durch Nutzer_innen hervorgebracht werden, die Offenbarung und Unterstützungssuche Betroffener massiv behindern können. Es spricht also einiges dafür, dass die Auseinandersetzung mit dieser Heuristik nicht nur für die medien- und sexualpädagogische Arbeit mit Heranwachsenden eine fruchtbare Perspektive bietet, sondern insbesondere auch eine Anforderung für die pädagogischen Fachkräfte selbst darstellt, die im Zuge gesellschaftlicher Mediatisierungsprozesse, der damit verbundenen Ambivalenzen und widersprüchlichen Gleichzeitigkeiten zunehmend unhintergehbar wird.

Eingegangen. 10. November 2021

Angenommen. 29. November 2021

Funding. Open Access funding enabled and organized by Projekt DEAL.

Open Access. Dieser Artikel wird unter der Creative Commons Namensnennung 4.0 International Lizenz veröffentlicht, welche die Nutzung, Vervielfältigung, Bearbeitung, Verbreitung und Wiedergabe in jeglichem Medium und Format erlaubt, sofern Sie den/die ursprünglichen Autor(en) und die Quelle ordnungsgemäß nennen, einen Link zur Creative Commons Lizenz beifügen und angeben, ob Änderungen vorgenommen wurden.

Die in diesem Artikel enthaltenen Bilder und sonstiges Drittmaterial unterliegen ebenfalls der genannten Creative Commons Lizenz, so- 


\section{Extrablick: Sexualität in der Sozialen Arbeit}

fern sich aus der Abbildungslegende nichts anderes ergibt. Sofern das betreffende Material nicht unter der genannten Creative Commons Lizenz steht und die betreffende Handlung nicht nach gesetzlichen Vorschriften erlaubt ist, ist für die oben aufgeführten Weiterverwendungen des Materials die Einwilligung des jeweiligen Rechteinhabers einzuholen.

Weitere Details zur Lizenz entnehmen Sie bitte der Lizenzinformation auf http://creativecommons.org/licenses/by/4.0/deed.de.

\section{Literatur}

Bauer Media Group (2016). Bravo Dr.-Sommer-Studie 2016. München: Bauer Media Group.

Böhm, M., \& Budde, J. (2020). Selbstbestimmt, konsensuell und wechselseitig? Perspektiven von Schüler*innen auf sexuelle Kommunikation mittels digitaler Medien am Beispiel ,Sexting'. Interdisziplinäre Fachzeitschrift für Prävention und Intervention, 23(1), 6-17.

Döring, N. (2011). Pornografie-Kompetenz: Definition und Förderung. In Zeitschrift für Sexualforschung, 24(3), 228-255.

Döring, N. (2015). Sexting. Aktueller Forschungsstand und Perspektiven für die Praxis. In Bundesarbeitsgemeinschaft Kinder- und Jugendschutz (Hrsg.), Gewalt im Netz. Sexting, Cybermobbing \& Co (S. 15-43). Berlin: Bundesarbeitsgemeinschaft Kinder- und Jugendschutz.

Gernert, J. (2010). Generation Porno. Jugend, Sex, Internet. Köln: Fackelträger Verlag.

Klein, A. (2014). Zwischen Online-Pornografie und Online-Beratung. Digitale Nutzungs- und Aneignungspraktiken Jugendliche. In J. Lauffer \& R. Roellecke (Hrsg.), Lieben, Liken, Spielen. Digitale Kommunikation und Selbstdarstellung Jugendlicher heute. Medienpädagogische Konzepte und Perspektiven. Beiträge aus Forschung und Praxis (S. 60-66). München: kopaed.

Klein, A. (2017). Verwahrlosung und Pornografie. Pädagogische und mediale Problematisierungen jugendlicher Sexualität. In A. Klein \& E. Tuider (Hrsg.), Sexualität und Soziale Arbeit (S. 83-101). Baltmannsweiler: Schneider Verlag Hohengehren.

Matthiesen, S. (2014). Was machen Jugendliche mit Internetpornografie? Ergebnisse einer Interviewstudie. In: K. Menne \& J. Rohloff (Hrsg.), Sexualität und Entwicklung: Beratung im Spannungsfeld von Normalität und Gefährdung (S.171-190). Weinheim: Juventa.

mpfs (2015). JIM-Studie 2020. Jugend, Information, (Multi-) Media. Basisuntersuchung zum Medienumgang 12- bis 19-Jähriger. https://www. mpfs.de/fileadmin/files/Studien/JIM/2015/JIM_Studie_2015.pdf. Zugegriffen: 9. Nov. 2021.

mpfs (2020). JIM-Studie 2020. Jugend, Information, Medien. Basisuntersuchung zum Medienumgang 12- bis 19-Jähriger. https://www.mpfs.de/fileadmin/files/Studien/JIM/2020/JIM-Studie-2020_Web_final.pdf. Zugegriffen: 9. Nov. 2021.

Ohlheiser, A. (2020). TikTok has become the soul of the LGBTQ internet. https://www.washingtonpost.com/technology/2020/01/28/tiktok-has-become-soul-lgbtq-internet/. Zugegriffen: 9. Nov. 2021.

Paus-Hasebrink, I. (2020). Medienkompetenz Herausforderungen im Umgang mit konvergierenden Medienangeboten. In J. Krone \& T. Pellegrini (Hrsg.), Handbuch Medienökonomie (S. 1217-1233). Wiesbaden: Springer VS.

Quandt, T., \& Vogelgesang, J. (2018). Jugend, Internet und Pornografie. In P. Rössler \& C. Rossmann (Hrsg.), Kumulierte Evidenzen. Replikationsstudien in der empirischen Kommunikationsforschung (S. 91-118). Wiesbaden: Springer VS.

Rack, S., \& Sauer, F. (2020). Selfies, Sexting, Selbstdarstellung. https:// www.klicksafe.de/fileadmin/media/documents/pdf/klicksafe_Materialien/ Lehrer_Always_On/KMA10_Selfies_Sexting_Selbstdarstellung_Mobile Medien_3.pdf. Zugegriffen: 9 . Nov. 2021.

Siller, F., Tillmann, A., \& Zorn, I. (2020). Medienkompetenz und medienpädagogische Kompetenz in der Sozialen Arbeit. In N. Kutscher, T. Ley, U. Seelmeyer, F. Siller, A. Tillmann \& I. Zorn (Hrsg.), Handbuch Soziale Arbeit und Digitalisierung (S. 315-333). Weinheim, Basel: Beltz Juventa.

Vogelsang, V. (2017). Sexuelle Viktimisierung, Pornografie und Sexting im Jugendalter: Ausdifferenzierung einer sexualbezogenen Medienkompetenz. Wiesbaden: Springer VS.

Hier steht eine Anzeige. 刑 Springer 
Hier steht eine Anzeige.

\section{Springer}

